\title{
LOCAL GAUGE AND MAGNETIC TRANSLATION GROUPS
}

\author{
W. FLOREK \\ Institute of Physics, Adam Mickiewicz University \\ Matejki 48/49, 60-769 Poznań, Poland
}

The magnetic translation group was introduced as a set of operators $T(\boldsymbol{R})=\exp [-\mathrm{i} \boldsymbol{R} \cdot(\boldsymbol{p}-e \boldsymbol{A} / c) / h]$. However, these operators commute with the Ilamiltonian for an electron in a periodic potential and a uniform magnetic field if the vector potential $\boldsymbol{A}$ (the gauge) is chosen in a symmetric way. It is showed that a local gauge field $A_{R}(r)$ on a crystal lattice leads to operators, which commute with the Hamiltonian for any (global) gauge field $\boldsymbol{A}=\boldsymbol{A}$ $(\boldsymbol{r})$. Such choice of the local gauge determines a factor system $\omega\left(\boldsymbol{R}, \boldsymbol{R}^{\prime}\right)=$ $T(R) T\left(R^{\prime}\right) T\left(R+R^{\prime}\right)^{-1}$, which depends on a global gauge only. Moreover, for any potential $A$ a commutator $T(R) T\left(R^{\prime}\right) T(R)^{-1} T\left(R^{\prime}\right)^{-1}$ depends only on the magnetic field and not on the gauge.

PACS numbers: 02.20.-a, 71.45.-d, 11.15.Ha, 05.50.+q

\section{Introduction}

The behavior of electrons in crystalline (periodic) potentials in the presence of a constant (external) magnetic field has been studied since the thirties in many papers (see, e.g., $[1,2]$ ). In the sixties Brown $[3]$ and Zak $[4,5]$ independently introduced and investigated the so-called magnetic translation groups. Their results have been lately applied to a problem of the quantum IIall effect $[6,7]$ and relations with the Weyl-IIeisenberg group have been also studied [8]. Some interesting results have been lately presented by Geyler and Popov [9].

The Hamiltonian for an electron in a periodic potential $V(r)$ and a uniform magnetic field (described by the vector potential $A$ ) is given as $[3,4]$

$$
\mathcal{H}=\pi^{2} / 2 m+V(\boldsymbol{r}), \text { where } \pi=\boldsymbol{p}+e \boldsymbol{A} / \boldsymbol{c}
$$

is the (vector) operator of the kinetic momentum. Brown introduced a projective representation of the translation group in the following form:

$$
T(\boldsymbol{R})=\exp [-\mathrm{i}(\boldsymbol{p}-e \boldsymbol{A} / \boldsymbol{c}) \cdot \boldsymbol{R} / \boldsymbol{h}] \text {. }
$$

These operators commute with Hamiltonian (1) if the vector potential $\boldsymbol{A}$ fulfills the following condition (see $[3,4]$ ):

$$
\partial A_{j} / \partial x_{k}+\partial A_{k} / \partial x_{j}=0 \text { for } j, k=1,2,3 \text {. }
$$


This relation holds, for example, for the gauge $A(r)=(H \times r) / 2$, which was used by Brown and Zak $[3,4]$. On the other hand, this condition is not satisfied by the Landau gauge $\boldsymbol{A}(\boldsymbol{r})=\left[-x_{2} H_{3}, 0,0\right]$ (for $\boldsymbol{H}=\left[0,0, H_{3}\right]$ ), which is used in many papers (e.g. [10]).

The aim of this paper is to find such a gauge $\boldsymbol{A}^{\prime}$ that: (i) $\nabla \times \boldsymbol{A}^{\prime}=-\boldsymbol{H}$; (ii) opcrators $T^{\prime}(\boldsymbol{R})=\exp \left[-\mathrm{i}\left(\boldsymbol{p}+\boldsymbol{A}^{\prime} c / c\right) \cdot \boldsymbol{R} / \hbar\right]$ commute with Hamiltonian (1); (iii) a factor system $\omega\left(\boldsymbol{R}, \boldsymbol{R}^{\prime}\right)=T(\boldsymbol{R}) T\left(\boldsymbol{R}^{\prime}\right) T\left(\boldsymbol{R}+\boldsymbol{R}^{\prime}\right)^{-1}$ depends only on a global gauge $A$, which defines the magnctic field (and the generalized momentum $\pi$ in (1)). It should be underlined that only the constant magnetic field $\boldsymbol{H}$ is considered. It occurs that these conditions are satisfied by a local gauge, i.e. an actual form of $\boldsymbol{A}^{\prime}(\boldsymbol{r})$ depends on a lattice vector $\boldsymbol{R}$.

\section{Solution}

For the constant magnetic ficld $\boldsymbol{H}=\left[I_{1}, I I_{2}, I I_{3}\right]$ the vector potential (gauge) $\boldsymbol{A}=\left[A_{1}, A_{2}, A_{3}\right]$ can be chosen as a linear function of $\boldsymbol{r}=\left[x_{1}, x_{2}, x_{3}\right]$ and can be written as

$$
A_{j}=\sum_{k=1}^{3} a_{j k} x_{k}, \quad \text { with } \quad a_{j k} \in \mathcal{R}, \quad a_{j j}=0 .
$$

Introducing a matrix $\mathcal{A}=\left(a_{j k}\right)$ it can be written as $\boldsymbol{A}=\mathcal{A} \boldsymbol{r}$. Therefore, the magnetic field $H$ is expressed by the matrix elements $a_{j k}$ as follows:

$$
I_{j}=-\sum_{k, l=1}^{3} \varepsilon_{j k l} a_{k l},
$$

which means that $\boldsymbol{H}$ is related to antisymmetrized matrix $\mathcal{A}$.

The definition (2) of operators $T(R)$ can be rewritten as

$$
T(R)=\exp \left(-\mathrm{i} \pi^{\prime} \cdot R / h\right)
$$

where

$$
\pi^{\prime}=p+e A^{\prime} / c \text { and } A^{\prime}(r)=-A(r),
$$

therefore $\nabla \times A^{\prime}=-\nabla \times \boldsymbol{A}=-\boldsymbol{H}$. Let us consider $\boldsymbol{A}^{\prime}=\boldsymbol{A}-\boldsymbol{H} \times \boldsymbol{r}=\mathcal{A}^{\mathrm{T}} \boldsymbol{r}$, i.e.

$$
A_{j}^{\prime}=A_{j}-\sum_{k, l=1}^{3} \varepsilon_{j k l} H_{k} x_{l}=\sum_{k=1}^{3} a_{k j} x_{k} .
$$

It is easy to note that $\nabla \times \boldsymbol{A}^{\prime}=-\boldsymbol{H}$. For example, assuming the Landau gauge (for $\left.\boldsymbol{H}=\left[0,0, H_{3}\right]\right)$ to be given as $\boldsymbol{A}=\left[-x_{2} H_{3}, 0,0\right]$ one obtains $\boldsymbol{A}^{\prime}=\left[0,-x_{1} H_{3}, 0\right]$, whereas the symmetric gauge $(H \times r) / 2$ yields $A^{\prime}=-(H \times r) / 2$. We have to check whether the operators $T(\boldsymbol{R})$ determined by the gauge $(7)$ commute with the IIamiltonian. It suffices to calculate commutators $\left[\pi_{j}, \pi_{k}^{\prime}\right]$ for $j, k=1,2,3$, for which one obtains

$$
\left[\pi_{j}, \pi_{k}^{\prime}\right]=\left[-\mathrm{i} \hbar \partial_{j}+e A_{j} / c,-\mathrm{i} \hbar \partial_{k}+e A_{k}^{\prime} / c\right]=-\mathrm{i} c h\left(\left[\partial_{j}, A_{k}^{\prime}\right]+\left[A_{j}, \partial_{k}\right]\right) / c=0 .
$$

To find a factor system of the above determined (projective) representations one has to calculate commutators $\left[X_{j} \pi_{j}^{\prime}, X_{k}^{\prime} \pi_{k}^{\prime}\right]\left(\boldsymbol{R}=\left[X_{1}, X_{2}, X_{3}\right]\right)$ :

$$
\begin{aligned}
& {\left[X_{j} \pi_{j}, X_{k}^{\prime} \pi_{k}^{\prime}\right]=X_{j} X_{k}^{\prime}\left[-\mathrm{i} \hbar \partial_{j}+e A_{j}^{\prime} / c,-\mathrm{i} \hbar \partial_{k}+e A_{k}^{\prime} / c\right]} \\
& \quad=-\mathrm{i} X_{j} X_{k}^{\prime} e \hbar / c\left(\partial_{j} A_{k}^{\prime}-\partial_{k} A_{j}^{\prime}\right)=-\mathrm{i} X_{j} X_{k}^{\prime} e h / c\left(a_{j k}-a_{k j}\right) .
\end{aligned}
$$

On the other hand, we have 
and, therefore

$$
\begin{aligned}
(\boldsymbol{R} & \left.\times \boldsymbol{R}^{\prime}\right) \cdot \boldsymbol{H}=-\sum_{l=1}^{3}\left(\sum_{j, k=1}^{3} \varepsilon_{l j k} X_{j} X_{k}^{\prime}\right)\left(\sum_{p, q=1}^{3} \varepsilon_{l p q} a_{p q}\right) \\
& =-\sum_{j, k=1}^{3} X_{j} X_{k}^{\prime}\left(a_{j k}-a_{k j}\right)
\end{aligned}
$$

$$
T(\boldsymbol{R}) T\left(\boldsymbol{R}^{\prime}\right)=T\left(\boldsymbol{R}+\boldsymbol{R}^{\prime}\right) \exp \left[-\mathrm{i}(e / h c)\left(\boldsymbol{R} \times \boldsymbol{R}^{\prime}\right) \cdot \boldsymbol{H} / 2\right] .
$$

It is interesting that this result does not depend on the chosen gauge $\boldsymbol{A}$ (and $\boldsymbol{A}^{\prime}$ ).

Let us consider now a local gauge determined as $A_{R}^{\prime}(r)=\mathcal{A}(r+R / 2)$, i.e.

$$
\left(A_{R}^{\prime}\right)_{j}(r)=\sum_{k=1}^{3} a_{k j}\left(x_{k}+X_{k} / 2\right)
$$

Similar, but a bit more tedious, calculations lead to the following results:

1. $\nabla \times A_{R}^{\prime}=-H$.

2. Operators $T(R)$ determined by this gauge commutc with Ilamiltonian (1).

3 . The projective representation $T(R)$ is characterized by a factor system

$\omega\left(\boldsymbol{R}, \boldsymbol{R}^{\prime}\right)=T(\boldsymbol{R}) T\left(\boldsymbol{R}^{\prime}\right) T\left(\boldsymbol{R}+\boldsymbol{R}^{\prime}\right)^{-1}=\exp \left[(-\mathrm{i} e / \hbar c)\left(\boldsymbol{R} \cdot \boldsymbol{A}\left(\boldsymbol{R}^{\prime}\right)\right)\right]$.

Note that a scalar product in the last equation can be also written as a bilinear form

$$
\boldsymbol{R} \cdot \boldsymbol{A}\left(\boldsymbol{R}^{\prime}\right)=\sum_{j, k=1}^{3} a_{j k} X_{j} X_{k}^{\prime}=\boldsymbol{R} \cdot \mathcal{A} \boldsymbol{R}^{\prime}=\mathcal{A}^{\mathrm{T}} \boldsymbol{R} \cdot \boldsymbol{R}^{\prime},
$$

i.e. it is fully determined by the matrix $\mathcal{A}$. It should be stressed that calculating $\omega\left(\boldsymbol{R}, \boldsymbol{R}^{\prime}\right)$ one has to take into account that

$$
T\left(\boldsymbol{R}+\boldsymbol{R}^{\prime}\right)=\exp \left[-\mathrm{i}\left(p+e \boldsymbol{A}_{\boldsymbol{R}}^{\prime} \boldsymbol{R}^{\prime} / c\right) \cdot\left(\boldsymbol{R}+\boldsymbol{R}^{\prime}\right) / \mathrm{h}\right] .
$$

The obtained factors (9) allow to find the commutator $T(R) T\left(R^{\prime}\right) T(R)^{-1} T\left(\boldsymbol{R}^{\prime}\right)^{-1}$ as

$$
\left.\omega\left(\boldsymbol{R}, \boldsymbol{R}^{\prime}\right) \omega\left(\boldsymbol{R}^{\prime}, \boldsymbol{R}\right)^{-1}=\exp \left[(-\mathrm{i} e / \hbar c) \boldsymbol{R} \cdot\left(\mathcal{A}-\mathcal{A}^{\mathrm{T}}\right) \boldsymbol{R}^{\prime}\right)\right] .
$$

Applying (10) this result can be also written as

$$
\exp \left[(-\mathrm{i} e / \hbar c)\left(\sum_{j, k=1}^{3} X_{j} X_{k}^{\prime}\left(a_{j k}-a_{k j}\right)\right)\right] \text {. }
$$

IIence, we have shown that

$$
T(\boldsymbol{R}) T\left(\boldsymbol{R}^{\prime}\right) T(\boldsymbol{R})^{-1} T\left(\boldsymbol{R}^{\prime}\right)^{-1}=\exp [(\mathrm{i} e / \hbar c) \Phi],
$$

where $\Phi=\left(\boldsymbol{R} \times \boldsymbol{R}^{\prime}\right) \cdot \boldsymbol{H}$ is a magnetic flux through the cell spanned by lattice vectors $\boldsymbol{R}$ and $\boldsymbol{R}^{\prime}$.

\section{Conclusion}

It was shown that a projective representation (6) of the translation group determined by the local gauge (8) has the following properties:

1. Operators $T(R)$ commute with IIamiltonian (1).

2. The factor system $\omega\left(\boldsymbol{R}, \boldsymbol{R}^{\prime}\right)$ depends on the global gauge $\boldsymbol{A}$, i.e. on the matrix $\mathcal{A}$.

3. The commutator of (magnetic) translations $T(\boldsymbol{R})$ and $T\left(\boldsymbol{R}^{\prime}\right)$ depends only on the magnetic field $\boldsymbol{H}$. 


\section{References}

[1] L. Landau, Z. Phys. 64, 629 (1930).

[2] R. Peierls, Z. Phys. 80, 763 (1933).

[3] E. Brown, Bull. Am. Phys. Soc. 8, 256 (1963); Phys. Rev. 133, A1038 (1964).

[4] J. Zak, Phys. Rev. 134, A1602, A1607 (1964).

[5] J. Zak, Phys. Rev. 136, A776 (1964).

[6] I. Dana, J. Zak, Phys. Rev. B 28, 811 (1983); Phys. Rev. B 32, 3612 (1985).

[7] I. Dana, Y. Avron, J. Zak, J. Phys. C, Solid State Phys. 18, L679 (1985).

[8] J. Zak, Phys. Lett. A 116, 195 (1986); Phys. Rev. B 39, 694 (1989).

[9] V.A. Geyler, I.Yu. Popov, Z. Phys. B 93, 437 (1994); Phys. Lelt. A 201, 359 (1995).

[10] P. Wiegmann, A. Zabrodin, Phys. Rev. Lett. 72, 1890 (1994). 